\title{
FEOCROMOCITOMA MALIGNO: COMUNICACIÓN DE 2 CASOS TRATADOS CON CIRUGÍA DE MÍNIMA INVASIÓN*
}

\author{
Drs. Octavio A. Castillo C. ${ }^{1,2}$, Pablo Riera A. ${ }^{3}$, Yáder Rodríguez J. ${ }^{1}$, \\ Enrique Alemán F. ${ }^{1}$, Gabriel Aranguren G. ${ }^{1}$, Jorge López-Vallejo C. ${ }^{1}$ \\ 1 Unidad de Urología y Centro de Cirugía Robótica, Clínica INDISA, Santiago. \\ 2 Facultad de Medicina, Universidad Andrés Bello, Santiago. \\ 3 Servicio de Urología, Hospital Gustavo Fricke, Viña del Mar. \\ Chile.
}

\begin{abstract}
Malignant pheochromocytoma treated with minimally invasive surgery

Introduction: Pheochromocytoma is a tumor derived from chromaffin cells that produces cathecolamines. Malignant pheochromocytomas are extremely rare. Aim: To describe 2 cases of malignant Pheochromocytoma treated with pure laparoscopic and robot assisted surgery. Clinical cases: Two female patients, 31 and 51 years of age, presented with a history of high blood pressure and abdominal pain. Radiologic and metabolic studies confirmed the diagnosis of Pheochromocytoma in both patients. A pure laparoscopic approach was performed in the first patient while a robotic assisted laparoscopy was done in the second patient. In both cases, adrenalectomy with in bloc nephrectomy was performed due to compromise of the renal sinus. There were no complications or conversion to open surgery. Histopathology confirmed the diagnosis of malignant Pheochromocytoma with metastasis to regional nodes and negative surgical margins. At follow-up, there is no evidence of recurrence. Conclusion: Malignant Pheochromocytoma can be treated with minimally invasive surgery, with morbidity and oncological outcomes similar to open approach.
\end{abstract} surgery.

Key words: Pheochromocytoma, malignant Pheochromocytoma, adrenal tumor, laparoscopy, robotic

\section{Resumen}

Introducción: El Feocromocitoma es un tumor de células cromafines productor de catecolaminas. Su variedad maligna es muy poco frecuente. Objetivo: Describir 2 casos de Feocromocitoma maligno tratados con cirugía mínimamente invasiva. Casos clínicos: Presentamos 2 pacientes de sexo femenino, de 31 y 51 años respectivamente. La primera con historia de hipertensión arterial de larga data y uso de múltiples fármacos. La segunda paciente debuta con dolor abdominal. En ambos casos los estudios radiológicos y meta-

*Recibido el 19 de marzo de 2013 y aceptado para publicación el 25 de abril de 2013.

Conflicto de Interés: Dr. Octavio A. Castillo se desempeña como Proctor de Latinoamérica para el robot da Vinci (Intuitive Surgical Inc.)

Correspondencia: Dr. Octavio A. Castillo C.

Av. Santa María 1810, Santiago, Chile. Código Postal: 7520440

octavio.castillo@indisa.cl 
bólicos confirmaron el diagnóstico de Feocromocitoma. A la primera paciente se le realizó un procedimiento laparoscópico puro, mientras que a la segunda paciente se le realizó un procedimiento laparoscópico asistido por robot. En ambos casos fue necesario realizar adrenalectomía en bloque con el riñón, por compromiso del hilio renal. No hubo complicaciones severas intra ni post-operatorias, como tampoco conversión a cirugía abierta. La histopatología confirmó el diagnóstico de Feocromocitoma maligno con metástasis ganglionares regionales y márgenes quirúrgicos negativos. Ambas se mantienen en control, sin evidencia de recurrencia. Conclusión: El Feocromocitoma maligno puede ser manejado de forma mínimamente invasiva, con morbilidad y resultados oncológicos comparables a cirugía abierta.

Palabras clave: Feocromocitoma, Feocromocitoma maligno, tumor adrenal, laparoscopía, cirugía robótica.

\section{Introducción}

El Feocromocitoma es una patología adrenal poco frecuente. Se trata de tumores productores de catecolaminas provenientes del tejido neuroectodérmico de la cresta neural. Suelen manifestarse en el contexto de síndromes familiares, como la Neurofibromatosis, Síndrome de Von Hippel Lindau, Síndrome de Sturge Weber, Esclerosis tuberosa y Neoplasias endocrinas múltiples (MEN) IIA y IIB ${ }^{1}$. Los Feocromocitomas malignos se comportan histológica y bioquímicamente igual que los benignos. De acuerdo a la Organización Mundial de la Salud (OMS), la malignidad de los Feocromocitomas se define por la presencia de metástasis a distancia, inclusive años después del diagnóstico y no por invasión local o por los sitios invadidos por células cromafínes $^{2}$. Se estima que $10-20 \%$ de los Feocromocitomas son malignos ${ }^{3}$. Si bien en algunos tumores existe un prominente grado de pleomorfismo nuclear, no existe ningún parámetro histológico que nos sirva para valorar su posible malignidad. Las manifestaciones clínicas dependen de la producción de catecolaminas, siendo la hipertensión arterial paroxística o constante el síntoma principal. Por lo tanto, el diagnóstico se basa en la sospecha clínica y la confirmación se realiza verificando la excreción aumentada de catecolaminas o sus metabolitos en orina de $24 \mathrm{~h}^{4}$. La localización del tumor se aprecia mediante Tomografía axial computada (TC) o Resonancia magnética (RM). El tratamiento es esencialmente quirúrgico, con extirpación completa de la masa. En los casos con metástasis o bordes quirúrgicos comprometidos se puede utilizar radio o quimioterapia, aunque solamente con intención paliativa ${ }^{5}$. En nuestro trabajo describimos nuestra experiencia en el manejo mínimamente invasivo del Feocromocitoma maligno.

\section{Casos clínicos}

\section{Caso clínico 1}

Paciente femenino, de 31 años de edad, con an- tecedente de hipertensión arterial de larga data en tratamiento con Carvedilol, Amlodipina, Doxazocina y Losartan. Refiere el antecedente de 2 cuadros de accidente cerebro-vascular, sin precisar fechas, resultando en una hemiparesia facio-braquio-crural izquierda.

En 2007 se realiza bypass gástrico por obesidad mayor de $300 \mathrm{~kg}$. Posteriormente, se realiza abdominoplastía en 2011, terminando con un índice de masa corporal de 37,6. En control rutinario se pesquisa una presión arterial de 190/119 por lo cual se realiza estudio de hipertensión secundaria. En TC de abdomen se evidencia una masa sólida, lobulada de 42 x $28 \mathrm{~mm}$, la cual reemplaza la glándula suprarrenal izquierda y adenopatías a nivel de los vasos renales izquierdos con englobamiento del hilio renal, de $78 \mathrm{~mm}$ (Figura 1). Sus exámenes de función suprarrenal mostraban Metanefrimas urinarias de 2.973 $\mathrm{ug} / 24 \mathrm{~h}$ y de $2.772 \mathrm{ug} / \mathrm{g}$ creatinina, Normetanefrinas de $21.505 \mathrm{ug} / 24 \mathrm{~h}$ y de $20.052 \mathrm{ug} / \mathrm{g}$ creatinina. Se realiza adrenalectomía más nefrectomía en bloque izquierda por vía laparoscópica (Figura 2). El tiempo quirúrgico fue de $120 \mathrm{~min}$ y el sangrado estimado fue de $1.000 \mathrm{ml}$. Durante la cirugía hubo 4 episodios de crisis hipertensiva moderada, fácilmente controladas con Nitroprusiato de Sodio endovenoso. No hubo complicaciones post-operatorias. El informe histopatológico reportó una masa suprarrenal de 9,2 x 8,5 x $5 \mathrm{~cm}$, compatible con Feocromocitoma maligno, con áreas de necrosis e infiltración hemorrágica y metástasis de 4 nodos linfáticos regionales. La inmunohistoquímica fue $(+)$ para Cromogranina y en más del 10\% de los núcleos para K167. La Citoqueratina fue negativa.

La paciente se mantiene en controles regulares, sin evidencia de recurrencia loco-regional o a distancia, a 12 meses de la cirugía.

\section{Caso clínico 2}

Paciente femenino de 51 años de edad, con antecedente de Artritis reumatoide en tratamiento. Tiene el antecedente de haberse realizado una abdominoplastía 30 días antes del ingreso. Dos días antes del 


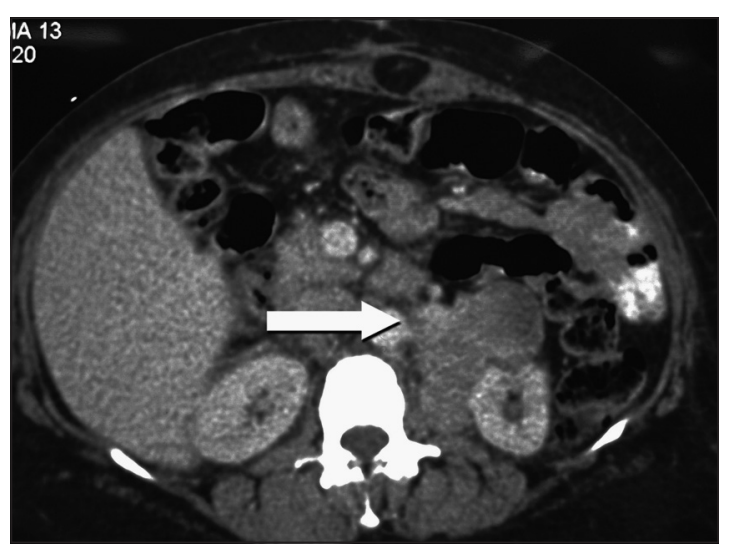

Figura 1. Tomografía computada que muestra masa tumoral heterogénea, comprometiendo la cara medial del riñón izquierdo.

ingreso presenta intenso dolor en flanco izquierdo, por lo cual se realiza Tomografía computada de abdomen la cual revela una masa de aspecto neoplásico en relación a la glándula suprarrenal izquierda, asociado a adenopatías para aórticas.

A su ingreso se encuentra una paciente enflaquecida, pálida. Su presión arterial es de 158/92, con una PAM de 118 y frecuencia cardíaca de $100 \mathrm{x}$ min. La paciente presentaba dolor intenso a la palpación en flanco izquierdo sin masa palpable. Ante la sospecha clínica de Feocromocitoma, se realizan pruebas hormonales, las cuales demuestran Norepinefrina plasmática de $8.247 \mathrm{ug} / \mathrm{ml}$, Normetanefrinas urinarias de $2.466 \mathrm{ug} / 24 \mathrm{~h}$ y Normetanefrinas 2.066 ug/g Creatinina. Las catecolaminas en orina reportaron Epinefrina de 12,6 ug/24 h y Norepinefrina en $1.541 \mathrm{ug} / 24 \mathrm{~h}$.

La RM confirma la presencia de una masa suprarrenal izquierda, heterogénea, de aproximadamente $5 \mathrm{~cm}$. La misma compromete la crura diafragmática, engloba el tronco celíaco, contacta la aorta y compromete el hilio renal. Se aprecian además varios linfonodos patológicos para-aórticos en relación al hilio renal (Figura 3).

Se realiza adrenalectomía más nefrectomía en bloque izquierda laparoscópica asistida por Robot, con disección completa de ambos órganos y liberación de la masa que engloba los vasos del hilio renal, arteria mesentérica superior y tronco celíaco. Durante la cirugía la paciente presentó 4 episodios de aumento de cifras tensionales, con un máximo de 160/90, que no ameritó el uso de Nitroprusiato de sodio endovenoso. El post-operatorio cursó sin incidentes.

El informe histopatológico reportó glándula suprarrenal de 5 x 4,5 cm y $52 \mathrm{~g}$ de peso con neo-

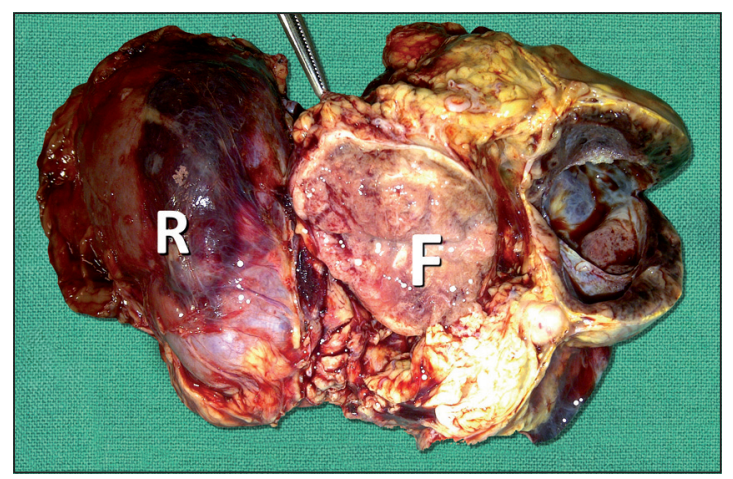

Figura 2. Fotografía macroscópica de masa tumoral de paciente 1. (R: riñón, F: feocromocitoma).

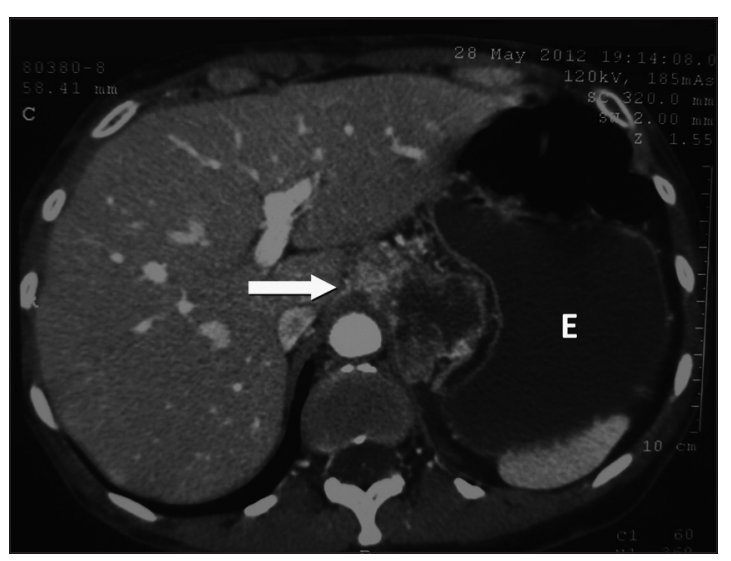

Figura 3. Resonancia magnética que muestra masa tumoral en región suprarrenal izquierda, la cual desplaza el estómago.

plasia epiteloide y extensas áreas de necrosis con extensión al tejido adiposo periadrenal y muscular estriado y permeaciones vasculares focales con un recuento mitótico de 4/10 campos de aumento mayor. Los márgenes quirúrgicos fueron negativos. La inmunohistoquímica fue $(+)$ para Synaptoficina en las células neoplásicas. Hubo 2/30 nodos linfáticos con metástasis de Feocromocitoma maligno en el tejido adiposo periadrenal e hilio renal.

La paciente ha completado 8 meses de control sin evidencias de recidiva tumoral.

\section{Discusión}

La evolución maligna de los Feocromocitomas depende fundamentalmente del trasfondo genético, describiéndose un alto riesgo de malignidad en tumores causados por mutación en el gen de la 
subunidad Beta de la Succinato Deshidrogenasa (SDHB). El único criterio de malignidad es la presencia de tejido cromafín no conectado al tumor primario, localizándose donde normalmente no debiera existir este tejido. Debido a este hecho, al diagnosticar un Feocromocitoma maligno, ya no existe la posibilidad de curar al paciente mediante cirugía. Por lo tanto, la cirugía se utiliza de forma paliativa para disminuir la masa tumoral y secreción de catecolaminas ${ }^{4}$. El pronóstico de estos pacientes es difícil de establecer, habiéndose comunicado una sobrevida a 5 años de $50 \%$ después de la cirugía. Existe consenso en que la resección quirúrgica es la primera opción, siempre y cuando sea posible. Sin embargo, existen otras modalidades terapéuticas como la administración de MIBG I ${ }^{131}$, quimioterapia o embolización arterial ${ }^{6}$. En un estudio prospectivo realizado por Aberbuch utilizando Ciclofosfamida, Vincristina y Dacarbazina, se obtuvo una respuesta radiológica en el $57 \%$ de los casos, respuesta hormonal en el $79 \%$ de los casos y una tasa media de supervivencia de 22 meses?

En cuanto a la administración de dosis variables de MIBG I ${ }^{131}$ con fines terapéuticos, Loh et al, realizaron una revisión de 116 pacientes, tras lo que se consiguió una respuesta radiológica parcial o completa en $30 \%$ de los casos, respuesta hormonal en $45 \%$ de los casos y una tasa media de supervivencia de 22 meses $^{8}$. Así mismo, Arias et al, reportaron un caso de Feocromocitoma maligno que recidivó 2 años después de la adrenalectomía. Doce años posterior a la segunda intervención quirúrgica, se presentó con metástasis ósea a nivel de la vértebra D11 confirmada con biopsia, por lo cual se inició terapia con MIBG $\mathrm{I}^{131}$ obteniendo una respuesta hormonal completa y una respuesta tumoral parcial $^{3,9}$. Por otro lado, Peñalver et al, utilizaron radioterapia paliativa para el control del dolor por metástasis óseas en un caso y posteriormente utilizaron MIBG I I31 para control de la extensión tumoral obteniendo una pobre respuesta y toxicidad grave al tratamiento $^{10,11}$. Hasta la fecha, nuestros 2 casos no han presentado sintomatología sugestiva de recidiva del Feocromocitoma. Sin embargo, el tiempo de seguimiento es corto, por lo que mantenemos una conducta expectante ante la posibilidad inminente de metástasis, inclusive a largo plazo. La técnica laparoscópica asistida por robot, si bien es una técnica relativamente nueva en el campo urológico, ha demostrado ser una opción válida para el tratamiento de los tumores suprarrenales. Treiyer et al, en una serie de 18 adrenalectomías laparoscópicas asistidas por robot, demostraron resultados equiparables a la cirugía abierta, con menores tasas de complicaciones intra y postoperatorias y menor estadía hospitalaria sin comprometer los resultados oncológicos ${ }^{12}$.

\section{Conclusión}

El Feocromocitoma maligno es una enfermedad agresiva y poco frecuente la cual es muy heterogénea en cuanto a su presentación clínica y sobrevida. No cabe duda que la intervención quirúrgica es el tratamiento inicial en el abordaje de esta enfermedad, siempre y cuando sea posible. La cirugía laparoscópica pura o asistida por robot es una opción factible en centros con experiencia. Debido a la agresividad de esta patología, debemos mantener una conducta expectante ante la posibilidad de recidivas y auxiliarnos en los diversos tratamientos existentes aunque sea de forma paliativa.

\section{Referencias}

1. Giménez Bachz JM, Salinas Sánchez AS, Lorenzo Romero JG, Segura Martin M, Hernández Millán I, Barba Romero MA. Feocromocitoma del órgano de Zuckerkandl. A propósito de un caso. Actas Urol Esp. 2002;26:372-76.

2. López Ferrandis J, Saiz Sansi A, Cervera Delgado M, Rioja Zuazu J, Regojo Balboa JM, Berian Polo JM. Feocromocitoma en Vena Cava. Actas Urol Esp. 2005;29:281-6.

3. Arias Martínez N, Barbado Hernández FJ, Couto Caro R, Gil Guerrero L, Coronado Poggio M, Navarro T, et al. Feocromocitoma maligno tratado con MIBG I ${ }^{131}$. An Med Intern. 2003; 20:575-8.

4. Scholz T, Eisenhofer G, Pacak K, Dralle H, Lehnert H. Clinical review: Current treatment of malignant pheochromocytoma. J Clin Endocrinol Metab. 2007;92:1217-25.

5. Chrisoulidou A, Kaltsas G, Ilias I, Grossman AB. Diagnosis and management of malignant pheochromocytoma and paraganglioma. Endocr Relat Cancer. 2007;14:56985.

6. Virseda Rodríguez JA, Martínez Ruiz J, Carrión López P, Martínez Sánchiz C, Donate Moreno MJ, Pastor Navarro H. Feocromocitoma: Más de una década de experiencia. Revisión de la literatura. Actas Urol Esp. 2010;34: 888-92.

7. Huang H, Abraham J, Hung E, Aberbuch, S, Merino M, Steinberg SM. Treatment of malignat pheochromocytoma/paraganglioma with cyclosphosphamide, vincristine, and dacarbazine: recommendation from a 22year follow-up of 18 patients. Cancer 2008;113:20208.

8. Loh KC, Fitzgerald PA, Matthay KK, Yeo PP, Price DC. The treatment of malignant pheochromocytoma with iodine-131 metaiodobenzylguanidine (131I-MIBG): a comprehensive review of 116 reported patients. J Endocrinol Invest. 1997;20:648-58.

9. Shou-Hung Tang, Ann Chen, Chun-Te Lee, Dah-Shyong 
Yu, Sun-Yran Chang, Gung-Huan Sun. Remote recurrence of malignant pheochromocytoma 14 years after primary operation. J Urol. 2003;169:269.

10. Peñalver D, Pavón De Paz I, Montoya T, MonereoMegias S. Feocromocitoma con metástasis vertebral. Endocrinol Nutr. 2004;51:557-61.

11. Real JT, Sánchez P, Catalá M, Lorente R, Ascaso JF,
Carmena R. Pancitopenia en un varón de 59 años con Feocromocitoma maligno tratado con I-131 metaiodobencilguanidina. Endocrinol Nutr. 2002;49:168-71.

12. Treiyer A, Janssen M, Kamradt J, Siemer S, Stockle M. Experiencia inicial con una serie de adrenalectomía laparoscópica asistida por robot. Actas Urol Esp. 2013;37:54-9. 